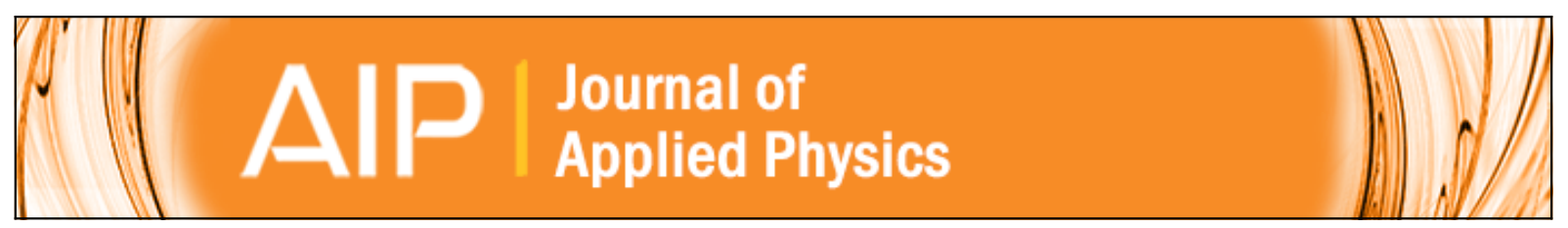

\title{
Ferromagnetism in Gd doped ZnO nanowires: A first principles study
}

S. Assa Aravindh, Udo Schwingenschloegl, and Iman S. Roqan

Citation: Journal of Applied Physics 116, 233906 (2014); doi: 10.1063/1.4904860

View online: http://dx.doi.org/10.1063/1.4904860

View Table of Contents: http://scitation.aip.org/content/aip/journal/jap/116/23?ver=pdfcov

Published by the AIP Publishing

\section{Articles you may be interested in}

Defect-band mediated ferromagnetism in Gd-doped $\mathrm{ZnO}$ thin films

J. Appl. Phys. 117, 013913 (2015); 10.1063/1.4905585

Size effects on formation energies and electronic structures of oxygen and zinc vacancies in $\mathrm{ZnO}$ nanowires: A first-principles study

J. Appl. Phys. 109, 044306 (2011); 10.1063/1.3549131

Indium-doped $\mathrm{ZnO}$ nanowires: Optical properties and room-temperature ferromagnetism

J. Appl. Phys. 108, 043516 (2010); 10.1063/1.3464229

Magnetic coupling properties of rare-earth metals (Gd, Nd) doped ZnO: First-principles calculations

J. Appl. Phys. 106, 023910 (2009); 10.1063/1.3176490

Magnetic coupling properties of Mn-doped $\mathrm{ZnO}$ nanowires: First-principles calculations

J. Appl. Phys. 103, 073903 (2008); 10.1063/1.2903332

MIT LINCOLN

LABORATORY CAREERS

Discover the satisfaction of innovation and service

to the nation
- Space Control

- Air \& Missile Defense

- Communications Systems \& Cyber Security

- Intelligence, Surveillance and

Reconnaissance Systems

- Advanced
Electronics
- Tactical Systems
- Homeland
Protection
- Air Traffic Control

LINCOLN LABORATORY

MassachusetTs Institute of TeChNOLOgY

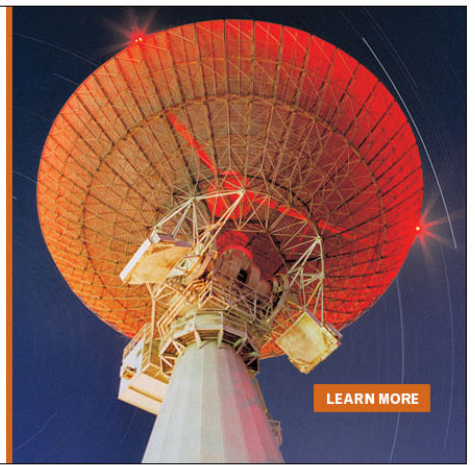




\title{
Ferromagnetism in Gd doped ZnO nanowires: A first principles study
}

\author{
S. Assa Aravindh, Udo Schwingenschloegl, ${ }^{\text {a) }}$ and Iman S. Roqan ${ }^{\text {a) }}$ \\ Physical Sciences and Engineering Division, King Abdullah University of Science and Technology (KAUST), \\ Thuwal 23955-6900, Kingdom of Saudi Arabia
}

(Received 17 September 2014; accepted 9 December 2014; published online 19 December 2014)

\begin{abstract}
In several experimental studies, room temperature ferromagnetism in $\mathrm{Gd}$-doped $\mathrm{ZnO}$ nanostructures has been achieved. However, the mechanism and the origin of the ferromagnetism remain controversial. We investigate the structural, magnetic, and electronic properties of $\mathrm{Zn}_{48} \mathrm{O}_{48}$ nanowires doped with $\mathrm{Gd}$, using density functional theory. Our findings indicate that substitutionally incorporated $\mathrm{Gd}$ atoms prefer occupying the surface $\mathrm{Zn}$ sites. Moreover, the formation energy increases with the distance between Gd atoms, signifying that no Gd-Gd segregation occurs in the nanowires within the concentration limit of $\leq 2 \%$. Gd induces ferromagnetism in $\mathrm{ZnO}$ nanowires with magnetic coupling energy up to $21 \mathrm{meV}$ in the neutral state, which increases with additional electron and $\mathrm{O}$ vacancy, revealing the role of carriers in magnetic exchange. The potential for achieving room temperature ferromagnetism and high $T_{C}$ in $\mathrm{ZnO}: \mathrm{Gd}$ nanowires is evident from the large ferromagnetic coupling energy $(200 \mathrm{meV})$ obtained with the $\mathrm{O}$ vacancy. Density of states shows that Fermi level overlaps with $\mathrm{Gd} f$ states with the introduction of $\mathrm{O}$ vacancy, indicating the possibility of $s-f$ coupling. These results will assist in understanding experimental findings in Gddoped $\mathrm{ZnO}$ nanowires. (C) 2014 AIP Publishing LLC. [http://dx.doi.org/10.1063/1.4904860]
\end{abstract}

\section{INTRODUCTION}

Recent years have witnessed a renewed research interest in $\mathrm{ZnO}$ due to its potential applications in optoelectronics, photochemical cells, diluted magnetic semiconductors (DMS), field effect transistors, and photoluminescent devices, to name a few. ${ }^{1-4}$ In searching for room temperature ferromagnetism (RTFM) in $\mathrm{ZnO}$, transition metal (TM) doping is widely investigated. ${ }^{5}$ Recently, the focus has shifted to rare-earth (RE) dopants, aiming to invoke magnetic coupling in $\mathrm{ZnO}$ by induced spin polarization. A few theoretical studies have reported weak FM in RE-doped bulk $\mathrm{ZnO}$ in zincblende and wurtzite structures. ${ }^{6-8}$ Even though the $4 f$ levels are localized in RE elements, $f$ electrons can couple strongly with host $s$ electrons, ${ }^{6}$ yielding the possibility of carriermediated $\mathrm{FM}^{7}{ }^{7}$ In particular, the interest in $\mathrm{ZnO}$ nanostructures has significantly increased in recent years owing to their unique optical, magnetic, and mechanical properties, leading to many potential applications. However, the mechanism and the origin of the ferromagnetism remain controversial. Assadi et al. ${ }^{7}$ have found that Eu ions doped into $\mathrm{ZnO}$ couple ferromagnetically when they are in nearest neighbor positions. While the same applies to Gd doping, the observed ferromagnetic coupling depends on the crystal structure and positions of the dopant atoms in the host matrix, as well as the distance between RE dopants. ${ }^{8}$ FM is observed experimentally in $\mathrm{ZnO}$ thin films ${ }^{9,10}$ and $\mathrm{ZnO}$ nanostructures doped with RE elements, such as Eu, Nd, and Gd. ${ }^{11,12}$ In several experiments on $\mathrm{ZnO}$ nanowires doped with $\mathrm{Gd}$, huge magnetic moments $\left(3278 \mu_{\mathrm{B}} / \mathrm{Gd}\right)$ were obtained, which is attributed to the exchange interaction between $4 f$ and $6 s$ electrons. ${ }^{11} \mathrm{Nd}$-doped $\mathrm{ZnO}$ nanowire arrays exhibit stable RTFM with magnetic

\footnotetext{
a) Authors to whom correspondence should be addressed. Electronic addresses: udo.schwingenschloegl@kaust.edu.sa and iman.roqan@kaust.edu.sa
}

moments of $4.1 \mu_{\mathrm{B}} / \mathrm{Nd}^{12}{ }^{12}$ Gd-implanted $\mathrm{ZnO}$ single crystals ${ }^{13}$ showed saturation moments of up to $1.8 \mu_{\mathrm{B}} / \mathrm{Gd}$ and $\mathrm{FM}$ has also been reported in nano-crystalline $\mathrm{ZnO}$ doped with $3.5 \% \mathrm{Gd}^{14}$ However, it has been noted that a high concentration of $\mathrm{Gd}$ impurities in $\mathrm{ZnO}$ thin films leads to paramagnetism. ${ }^{15}$

In this scenario, doping with Gd seems to be a promising way to achieve RTFM in $\mathrm{ZnO}$ nanostructures. However, the existing experimental results are controversial and depend strongly on the doping method and the defect type. ${ }^{10-12,15}$ In addition, the mechanism and origin of the magnetic coupling are poorly understood. The few theoretical studies that have been conducted thus far deal with RE doping in bulk $\mathrm{ZnO}$ and do not elucidate the mechanisms responsible for RTFM in Gd-doped wurtzite $\mathrm{ZnO}$ nanostructures. ${ }^{6-8}$ On the other hand, it is important to understand the physical mechanisms responsible for the FM in $\mathrm{ZnO}$ nanostructures from both fundamental and technological perspectives. In this paper, we demonstrate $\mathrm{FM}$ in $\mathrm{Gd}$-doped $\mathrm{Zn}_{48} \mathrm{O}_{48}$ nanowires oriented along the wurtzite [0001] direction, as well as the effect of point defects on the magnetic coupling, using first-principles calculations. As it is not computationally feasible to model nanostructures beyond a few nanometers, density functional theory (DFT) studies are often conducted on nanowires of diameters ranging from sub-nanometer to $\leq 3 \mathrm{~nm}$ in order to study the properties of nanowires and allow comparisons with experimental findings. ${ }^{16,17}$ In particular, nanowires of diameter ranging from 1.6 to $5 \mathrm{~nm}$ can now be fabricated by experimental techniques. ${ }^{18}$ In addition, DFT studies on nanowires of diameter less than $1 \mathrm{~nm}$ assist in understanding the optical properties due to the presence of quantum size effects that generally result in substantial density of states (DOSs) at the band edge. ${ }^{17}$ The diameter of the wurtzite $\mathrm{ZnO}$ nanowire in the present study is about $1 \mathrm{~nm}$, which is sufficient for examining the relative stability and segregation of $\mathrm{Gd}$, in the 
dilute limit. In order to advance the understanding of the magnetic mechanism, we also investigate the electronic structure of these nanowires.

\section{COMPUTATIONAL METHODOLOGY}

In this work, a $\mathrm{Zn}_{48} \mathrm{O}_{48}$ nanowire of about $1 \mathrm{~nm}$ diameter, oriented along wurtzite [0001] direction is modeled using the supercell approach. A vacuum of $15 \AA$ thickness is created along $\mathrm{X}$ and $\mathrm{Y}$ directions to ensure that the interaction between the nanowire and its periodic images remains negligible. The supercell has a length of $2 c$, where $c$ is the bulk lattice parameter along the [0001] direction, such that it contains 96 atoms $\left(\mathrm{Zn}_{48} \mathrm{O}_{48}\right)$. We employ the Vienna $A b$ initio Simulation Package $(\mathrm{VASP})^{19}$ to carry out our spinpolarized first-principles simulations, using the generalized gradient approximation (GGA) ${ }^{20}$ and plane wave cut-off energy of $400 \mathrm{eV}$. Methods such as GGA+U and SelfInteraction Corrected LDA (SIC-LDA) ${ }^{21}$ are widely used to treat $\mathrm{ZnO}$-based systems, as this allows overcoming the "band-gap" problem. A comparison of the results pertaining to $\mathrm{LDA}$ and $\mathrm{LDA}+\mathrm{U}$ in $\mathrm{ZnO}: \mathrm{Gd}$ systems showed that the qualitative picture is mostly unchanged, with the exception of some quantitative changes. ${ }^{6,22,23}$ Hence, we do not expect the main trend of electron-mediated FM described in our paper to be altered significantly if Hubbard $U$ correction or SIC-LDA is taken into account. The pseudopotentials are based on the projector augmented wave formalism. ${ }^{24}$ The Brillouin zone sampling is carried out in the MonkhorstPack scheme using a $1 \times 1 \times 8$ mesh. The atomic coordinates are allowed to relax without geometry and spin constraints. Energy and force tolerances of $0.0001 \mathrm{eV}$ and $0.004 \mathrm{eV} / \AA$, respectively, are achieved.

The $\mathrm{Gd}$ atoms are incorporated into $\mathrm{Zn}_{48} \mathrm{O}_{48}$ nanowires at substitutional $\mathrm{Zn}$ sites, which are favored by $\mathrm{RE}$ elements. ${ }^{8}$ To determine the preferred Gd positions, total energy calculations are performed for 12 configurations, divided into 3 groups, encompassing surface, sub-surface, and bulk-like regions, as shown in Fig. 1 (groups 1, 2, and 3, respectively). These calculations are conducted on freestanding nanowires without surface passivation. The formation energy $\left(E^{f}\right)$ of $\mathrm{Gd}$ is calculated as

$$
E^{f}=E\left(\mathrm{Zn}_{48-\mathrm{m}} \mathrm{O}_{48} \mathrm{Gd}_{\mathrm{m}}\right)-E\left(\mathrm{Zn}_{48} \mathrm{O}_{48}\right)+n \mu(\mathrm{Zn})-m \mu(\mathrm{Gd}),
$$

where $E$ and $\mu$ represent the total energy and chemical potential (total energy of metallic $\mathrm{Zn}$ and Gd), respectively. Here, $n$ is the number of $\mathrm{Zn}$ atoms removed from the supercell and $m$ denotes the number of $\mathrm{Gd}$ atoms substituted to the $\mathrm{Zn}$ sites.

\section{RESULTS AND DISCUSSION}

We first optimize the internal coordinates of the atoms in the nanowires without dopant. The relaxation of atomic coordinates is important in the pristine nanowire, due to the large surface area/volume ratio. We find that the relaxation of the surface atoms is larger than that of the inner atoms. In bulk wurtzite $\mathrm{ZnO}$, the $\mathrm{Zn}-\mathrm{O}$ bond length is $1.99 \AA$. For the relaxed nanowire, this bond length becomes $1.89 \AA$ in the $c$-direction, while it is $2.06 \AA$ in the $a b$-plane. Therefore, after optimization, the nanowires have distortion along the $c$ axis, compared to the bulk.

One $\mathrm{Gd}$ atom is substitutionally incorporated to the $\mathrm{Zn}$ site in the $\mathrm{Zn}_{48} \mathrm{O}_{48}$ nanowire (corresponding to a doping concentration of about $1 \%$ ) and relaxation is carried out without any geometry or spin constraints. We consider all symmetrically non-equivalent substitutional sites, as shown in Fig. 1. We find that the Gd-O bond length along the $c$-direction changes from $1.89 \AA$ to $2.08 \AA$, while the change within the $a b$-plane is negligible $(0.01 \AA)$. Hence, the $\mathrm{Zn}_{47} \mathrm{O}_{48} \mathrm{Gd}$ nanowires reach structural stability with less pronounced lattice distortion. The calculated $E^{f}$ of $\mathrm{Gd}$ atoms in the $\mathrm{Zn}_{47} \mathrm{O}_{48} \mathrm{Gd}$ nanowires is also given in Fig. 1. The formation energy in the neutral state determines the solubility in the host matrix, with a high value indicating low solubility.

The lowest value of $E^{f}$ (favorable position) is obtained when the Gd atom is located on the surface of the nanowire (group 1c). Correspondingly, the value of $E^{f}$ increases as the Gd location shifts towards the bulk-like region. This preference of the surface sites is in line with the experimental
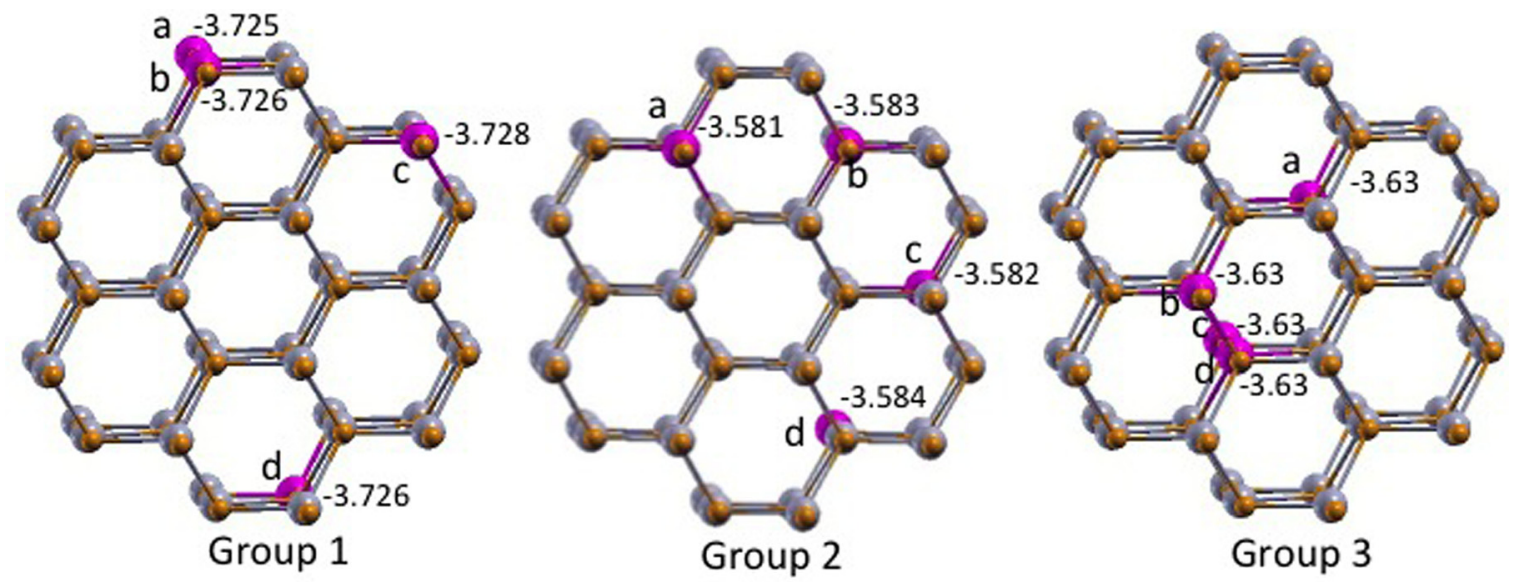

FIG. 1. $\mathrm{Zn}_{47} \mathrm{O}_{48} \mathrm{Gd}$ nanowire configurations with one $\mathrm{Zn}$ atom replaced by Gd. The 12 configurations are divided into 3 groups for all non-equivalent substitutional sites. The energy (in eV) is denoted for each configuration. 
findings in which RE dopants accumulate on the surface of $\mathrm{ZnO}$ nanowires during in situ deposition. ${ }^{11} \mathrm{We}$ find that an isolated $\mathrm{Gd}$ atom incorporated in a $\mathrm{Zn}$ site exhibits a magnetic moment of about $6.87 \mu_{\mathrm{B}}$, for all Gd positions.

Increasing the $\mathrm{Gd}$ concentration will provide insight into the energetic preference, as well as about the Gd-Gd interaction, and allow us to gain better understanding of the potential for RTFM. For this purpose, we incorporate two $\mathrm{Gd}$ atoms into the $\mathrm{Zn}_{48} \mathrm{O}_{48}$ nanowire ( $\mathrm{Gd}$ concentration of about $2 \%$ ), considering different configurations of substitutional sites in the surface, sub-surface, and bulk-like regions. In addition, for each Gd-Gd distance, several spatial arrangements are considered. The $E^{f}$ for all configurations is calculated using Eq. (1). Fig. 2 shows $E^{f}$ as a function of Gd-Gd distance in $\mathrm{Zn}_{46} \mathrm{O}_{48} \mathrm{Gd}_{2}$ nanowire. As the $\mathrm{Gd}$ atoms prefer the surface substitutional $\mathrm{Zn}$ sites, we examine possible surface configurations and for comparison, the sub-surface and bulk-like regions. We obtain the lowest values of $\mathrm{E}^{f}$ for the surface configurations. Interestingly, for similar Gd-Gd distances, $E^{f}$ is lower at the surface than in the bulk-like region. In addition, Gd atoms prefer to stay away from each other (as shown in Fig. 2), indicating that no Gd segregation can occur for Gd-doped $\mathrm{ZnO}$ nanowire in the low concentration limit of this study. This finding implies that magnetism observed in $\mathrm{ZnO}$ nanostructures with low $\mathrm{RE}$ concentration ( $2 \%$, i.e., below the solubility limit) may not be due to $\mathrm{Gd}$ clusters. On the other hand, extant experimental studies have reported clustering tendency at higher RE concentrations $(>2.5 \%)$ in $\mathrm{ZnO}^{25}$ Several studies indicate that $\mathrm{ZnO}$ doped with TMs often suffers from the magnetic percolation problem at impurity concentrations of about 5\%-10\%, due to short-ranged exchange interactions. Therefore, obtaining high $T_{C}$ in $\mathrm{ZnO}$ doped with $\mathrm{RE}$ and TM is difficult due to the magnetic percolation issues. ${ }^{26}$ In this case, if the doped $\mathrm{ZnO}$ undergoes spinodal decomposition, due to the attractive interaction between magnetic clusters, magnetic percolation paths can be formed, leading to superparamagnetism, as a result of the strong magnetic coupling between atoms in the same cluster. If the impurity cluster is sufficiently large, the relaxation time required to flip the magnetization becomes

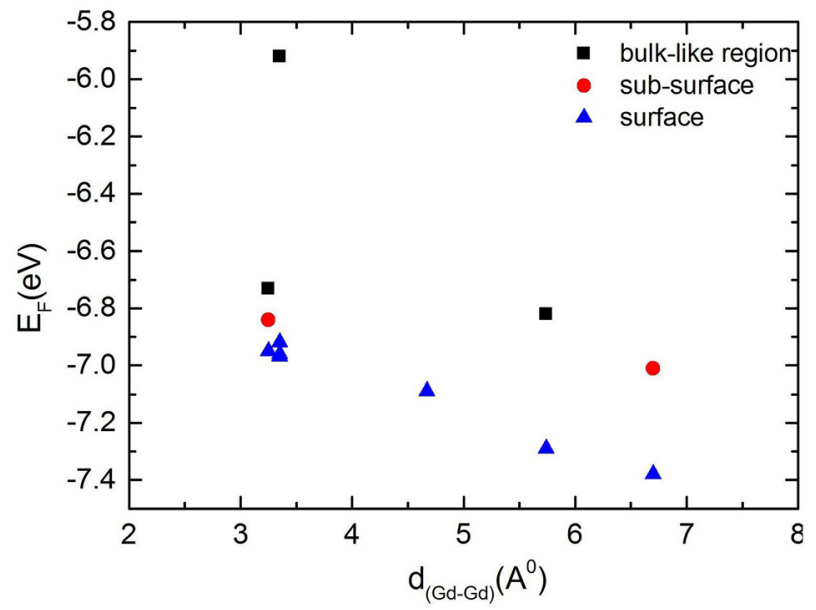

FIG. 2. The formation energy of $\mathrm{Gd}$ atoms in $\mathrm{Zn}_{46} \mathrm{O}_{48} \mathrm{Gd}_{2}$ nanowires as a function of the separation between two Gd atoms. rather long, enhancing the blocking temperature. However, the Gd concentrations considered in the present study $(\leq 2 \%)$ are too low to observe magnetic percolation effects. ${ }^{27}$

We investigate the possibility of exchange coupling between $\mathrm{Gd}$ atoms by calculating the energy difference $(\Delta \mathrm{E})$ between ferromagnetic and antiferromagnetic (AFM) (as shown in Table I and Fig. 3) configurations. In Figs. 3(a)-3(d), some selected configurations for different Gd substitutional sites on the surface are shown. These configurations can be contrasted with Figs. 3(e)-3(g), which show the nanowire configurations in which $\mathrm{Gd}$ atoms are substituted in the bulk-like region of the nanowire. We show this comparison because it has been reported that, during RE ion implantation into $\mathrm{ZnO}$, the dopant atoms are distributed homogeneously. ${ }^{28}$

We find that $\Delta \mathrm{E}$ is sufficiently large in favor of FM coupling in Gd-doped $\mathrm{ZnO}$ nanowires in the neutral state. A significant $\Delta \mathrm{E}$ (as high as $21 \mathrm{meV}$ ) is obtained for the nearest neighbor $\mathrm{Gd}$ atoms in the neutral state. However, the $\Delta \mathrm{E}$ value decreases to $4 \mathrm{meV}$ as the distance between $\mathrm{Gd}$ dopants increases (in defect-free $\mathrm{Zn}_{46} \mathrm{O}_{48} \mathrm{Gd}_{2}$ nanowire), as shown in the second column of Table I. Furthermore, we investigate the effects of additional charge injected into the nanowires. According to our findings, introduction of one electron clearly enhances the FM, as can be seen in the third column of Table I. We also introduce intrinsic point defects, such as $\mathrm{Zn}$ vacancy $\left(\mathrm{V}_{\mathrm{Zn}}\right)$ and $\mathrm{O}$ vacancy $\left(\mathrm{V}_{\mathrm{O}}\right)$. It has been shown previously that $\mathrm{V}_{\mathrm{O}}$ has the lowest $\mathrm{E}^{f}$ among the native donor defects in $\mathrm{ZnO}{ }^{29}$ The location of $\mathrm{V}_{\mathrm{O}}$ in each nanowire configuration is indicated in Fig. 3. We find that $V_{Z n}$ does not enhance the FM, whereas $V_{O}$ has a drastic effect, much stronger than the additional charge (see the fourth column of Table I). This phenomenon is due to the fact that, one $V_{O}$ introduces two electrons and leads the system to a more stable FM state. In the presence of $\mathrm{V}_{\mathrm{O}}, \Delta \mathrm{E}$ is significantly larger $(200 \mathrm{meV})$ than that measured for a defect-free $\mathrm{ZnO}$ nanowire. Hence, long-range FM can be achieved when the GdGd distance is maintained within $6 \AA$. This is in line with extended x-ray absorption fine structure measurements; RE dopants are found to be incorporated into $\mathrm{ZnO}$ nanowires at distances below $5 \AA .{ }^{25}$ The observed effects of the additional charge and $\mathrm{V}_{\mathrm{O}}$ support the premise that the FM is due to $f-s$ coupling. Experimental findings show that FM due to electron-mediated exchange is observed in n-type $\mathrm{ZnO},{ }^{30}$ in line with our calculated results. However, our findings

TABLE I. Total energy difference between FM and AFM configurations, for the $\mathrm{Zn}_{46} \mathrm{O}_{48} \mathrm{Gd}_{2}$ nanowires shown in Fig. 3 without defects (second column) and with the introduction of electron (e) (third column) and $V_{O}$ (fourth column).

\begin{tabular}{lccc}
\hline \hline Configuration & $\Delta \mathrm{E}(\mathrm{meV})$ & $\Delta \mathrm{E}(\mathrm{e})(\mathrm{meV})$ & $\Delta \mathrm{E}\left(\mathrm{V}_{\mathrm{O}}\right)(\mathrm{meV})$ \\
\hline $\mathrm{a}$ & 4 & 20 & 31 \\
$\mathrm{~b}$ & 11 & 35 & 96 \\
$\mathrm{c}$ & 21 & 57 & 134 \\
$\mathrm{~d}$ & 9 & 23 & 108 \\
$\mathrm{e}$ & 17 & 86 & 200 \\
$\mathrm{f}$ & 13 & 21 & 60 \\
$\mathrm{~g}$ & 9 & 14 & 46 \\
\hline \hline
\end{tabular}



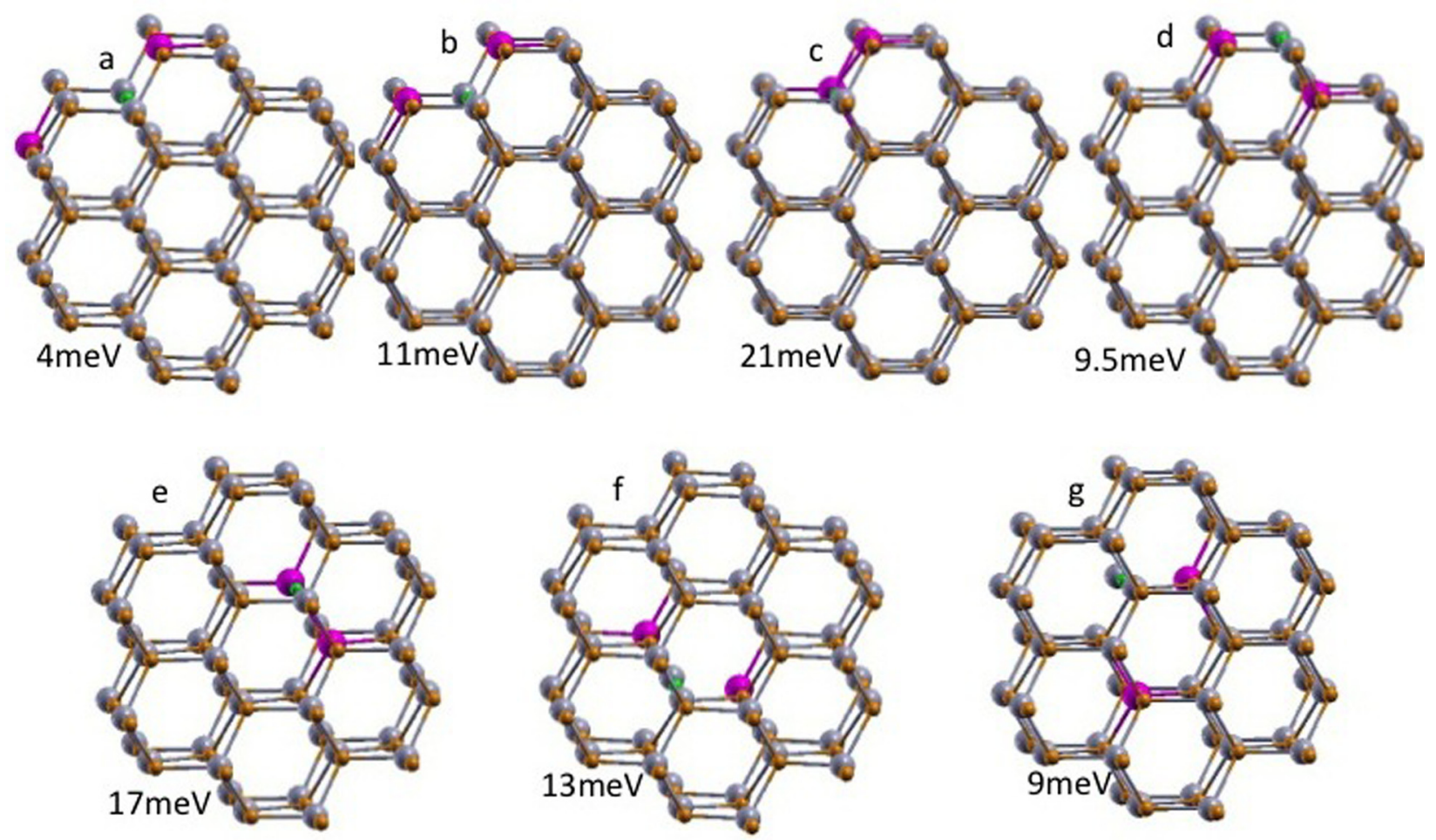

FIG. 3. Different configurations of $\mathrm{Zn}_{46} \mathrm{O}_{48} \mathrm{Gd}_{2}$ nanowires with two $\mathrm{Zn}$ atoms replaced by Gd. The total energy differences between FM and AFM for each configuration are shown. The $\mathrm{V}_{\mathrm{O}}$ positions are indicated by green spheres. Note that the FM coupling varies with the distance and location of Gd atoms in the nanowire.

suggest that RTFM can be stabilized in $\mathrm{ZnO}$ :Gd nanowires, by electrons and $\mathrm{V}_{\mathrm{O}}$, as $\mathrm{FM}$ is favored with a significant energy gain $(\Delta E>30 \mathrm{meV})$, which is sufficient to establish RTFM. This confirms that superparamagnetic phase cannot be occurred in these nanowires. In this study, we show that, due to the strong localized nature of the $4 f$ electrons, carriers are required to establish ferromagnetic exchange in REdoped $\mathrm{ZnO}$. On the other hand, in the case of TM-based $\mathrm{ZnO}$ materials, FM can be stabilized with or without carriers. ${ }^{30,31}$ The large $\Delta \mathrm{E}$ obtained in our investigation is an indication of the possibility of achieving long-range FM with high $T_{C}$ ( $>\mathrm{RT}$ ) in $\mathrm{RE}$ doped $\mathrm{ZnO}$ systems, since it is experimentally demonstrated that, with varying electron concentrations, high $T_{C}$ can be obtained in n-type $\mathrm{ZnO}: \mathrm{Gd}^{32}$

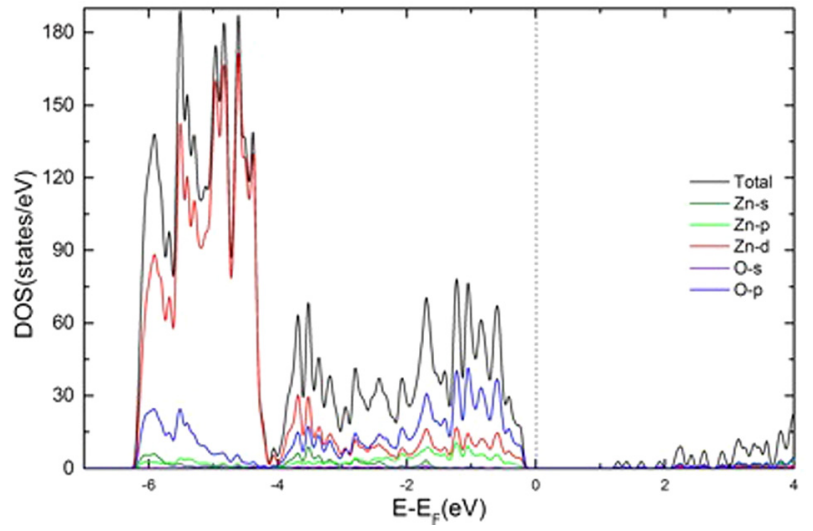

FIG. 4. The density of states of the pristine $\mathrm{Zn}_{48} \mathrm{O}_{48}$ nanowire without $\mathrm{Gd}$ dopants.
We also investigate the DOS, as it can provide further insight into the exchange mechanism. Fig. 4 depicts the DOS of pristine $\mathrm{Zn}_{48} \mathrm{O}_{48}$ nanowire, exhibiting a semiconducting and nonmagnetic nature, in line with previous calculations. ${ }^{33}$ The upper part of the valence band (from -4 to $0 \mathrm{eV}$ ) is mainly due to $\mathrm{O} 2 p$ states. However, the contribution of $\mathrm{Zn} d$ states becomes prominent in the $-4 \mathrm{eV}$ to $-6 \mathrm{eV}$ range, which is buried deeply inside the valence band. $\mathrm{Zn} 3 d$ and $\mathrm{O}$ $2 p$ states reveal an overlap between the electronic states, which results in strong hybridization. The $\mathrm{Zn}, \mathrm{O}$ and $\mathrm{Gd}$ DOSs for the $\mathrm{Zn}_{46} \mathrm{O}_{48} \mathrm{Gd}_{2}$ nanowire with and without $\mathrm{V}_{\mathrm{O}}$ are shown in Fig. 5, in the top and bottom panels, respectively.

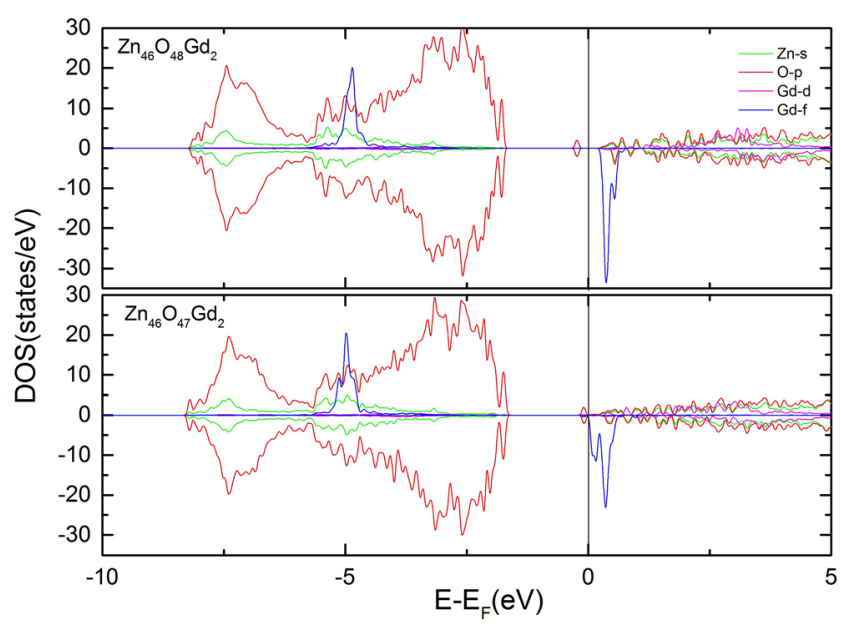

FIG. 5. The densities of states of the $\mathrm{Zn}_{46} \mathrm{O}_{48} \mathrm{Gd}_{2}$ and $\mathrm{Zn}_{46} \mathrm{O}_{47} \mathrm{Gd}_{2}$ nanowires (top and bottom panels), respectively. The projected density of states of $\mathrm{Zn}(s), \mathrm{O}(p), \mathrm{Gd}(d)$, and $\mathrm{Gd}(f)$. 
The spin-up and spin-down DOSs of doped nanowire are significantly different from that of the pristine nanowire. For $\mathrm{ZnO}$ doped with $\mathrm{Gd}$, a remarkable shift in the Fermi level into the conduction band, close to the $\operatorname{Gd} f$ states, is observed. The majority Gd $f$ states (spin up) that are located well below the valence band maximum are fully occupied. On the other hand, the minority unoccupied $\mathrm{Gd} f$ states are localized in the vicinity of the Fermi level. The Gd $d$ states in the conduction band overlap with the $\mathrm{Gd} f$-states. Hybridization of the Gd $f$ states with the states of the host material occurs at the Fermi level. As a result of introducing $\mathrm{V}_{\mathrm{O}}$, the $\mathrm{Gd} f$ state near the Fermi energy becomes partially occupied by donor electrons. Consequently, the carrier concentration around $\mathrm{V}_{\mathrm{O}}$ is increased, which mediates interaction between the $s$ (mostly from $\mathrm{Zn}$ ) and $f$ electrons. This is evident from the DOS, as the $s-f$ coupling is more prominent than $p-f$ and $f-f$ couplings. Since $d$ orbitals are not contributing to the partially filled energy bands, the double exchange picture is inappropriate for describing the exchange interaction in the current context of $\mathrm{ZnO}: \mathrm{Gd}$ nanowires. ${ }^{34}$ In this case, when Fermi level overlaps with the Gd $f$-state (which becomes partially filled) donor electrons occupy empty $f$ states and thus FM coupling occurs. ${ }^{23}$ The type of carriers involved in establishing long-range ferromagnetic order determines the exchange interactions in DMS materials. Zener's $p-d$ exchange mechanism, as described by Dietl et $a l .{ }^{1}$ is weaker and long-ranged, mediated by extended band of the valence hole states. The double-exchange model is based on a physical picture of the $d$ electron hopping between atoms with strong on-site exchange. ${ }^{34}$ In the present study of $\mathrm{ZnO}: \mathrm{Gd}$ nanowires, $\mathrm{V}_{\mathrm{O}}$ donates two electrons to the system, mediating the ferromagnetic exchange and hence, the $s-f$ coupling is more prominent than other mechanisms. ${ }^{34,35}$ According to our calculations, introducing $\mathrm{V}_{\mathrm{Zn}}$ does not increase the ferromagnetic coupling energy. Moreover, $p$ - $f$ exchange interaction mediated by holes is not possible, as the calculated DOS of Gd-doped $\mathrm{ZnO}$ nanowire shows a shift of Fermi level towards the conduction band, implying that electrons can mediate FM through $s-f$ interactions. Hence, it can be concluded that the increase in $\Delta \mathrm{E}$ (up to $200 \mathrm{meV}$ ) with the introduction of $\mathrm{V}_{\mathrm{O}}$ is due to this strong $s-f$ coupling. Thus, the presence of $\mathrm{V}_{\mathrm{O}}$ is crucial for increasing the $s-f$ coupling (as $4 f$ state is strongly localized) and therefore promotes long-range RTFM in Gd-doped $\mathrm{ZnO}$ nanowires.

\section{CONCLUSIONS}

A systematic study of $\mathrm{Gd}$ doping in $\mathrm{ZnO}$ nanowires showed that the energetically favorable substitutional sites are located on the surface of the nanowire, which is in agreement with previous experimental observations. We have shown that the exchange coupling between the Gd atoms is sufficient for establishing RTFM. Introduction of additional charge or $\mathrm{V}_{\mathrm{O}}$ increases the ferromagnetic coupling energy by enhancing the $s-f$ coupling significantly, as Fermi level is shifted further into the conduction band, close to the $\operatorname{Gd} f$ states. Our findings can be used to understand experimentally observed RTFM in Gd-doped ZnO nanowires.
${ }^{1}$ T. Dietl, H. Ohno, F. Matsukura, J. Cibert, and D. Ferrand, Science 287, 1019 (2000).

${ }^{2}$ P. Sharma, A. Gupta, K. V. Rao, F. J. Owens, R. Sharma, R. Ahuja, J. M. O. Guillen, B. Johansson, and G. A. Gehring, Nature Mater. 2, 673 (2003). ${ }^{3}$ A. Mang, K. Reimann, and S. Rubenacke, Solid State Commun. 94, 251 (1995). ${ }^{4}$ M. H. Huang, S. Mao, H. Feick, H. Q. Yan, Y. Y. Wu, H. Kind, E. Weber, R. Russo, and P. Yang, Science 292, 1897 (2001).

${ }^{5}$ U. Ozgur, Y. I. Alivov, C. Liu, A. Teke, M. A. Reshchikov, S. Doan, V. Avrutin, S.-J. Cho, and H. Morkoc, J. Appl. Phys. 98, 041301 (2005).

${ }^{6}$ H. Shi, P. Zhang, S. S. Li, and J. B. Xia, J. Appl. Phys. 106, 023910 (2009).

${ }^{7}$ M. H. N. Assadi, Y. Zhang, R. Zheng, S. P. Ringer, and S. Li, Nanoscale Res. Lett. 6, 357 (2011).

${ }^{8}$ I. Bantounas, S. Goumri-Said, M. B. Kanoun, A. Manchon, I. Roqan, and U. Schwingenschlogl, J. Appl. Phys. 109, 083929 (2011).

${ }^{9}$ P. Photongkam, Y. B. Zhang, M. H. N. Assadi, S. Li, D. Yu, M. Lonescu, and A. V. Pan, J. Appl. Phys. 107, 033909 (2010).

${ }^{10}$ X. Ma, Thin Solid Films 520, 5752 (2012).

${ }^{11}$ M. Ungureanu, H. Schmidt, Q. Xu, H. V. Wenckstern, D. Spemann, H. Hochmuth, M. Lorenz, and M. Grundmann, Superlattices Microstruct. 42, 231 (2007).

${ }^{12}$ D. Wang, Q. Chen, G. Xiang, J. Yi, S. Bakul, J. Dung, J. Wang, and T. Wu, Nano Lett. 12, 3994 (2012).

${ }^{13}$ K. Potzger, S. Zhou, F. Eichhorn, M. Helm, W. Skorupa, A. Mucklich, and J. Fassbender, J. Appl. Phys. 99, 063906 (2006).

${ }^{14}$ A. A. Dakhel and M. El-Hilo, J. Appl. Phys. 107, 123905 (2010).

${ }^{15}$ N. Ney, S. Ye, T. Kammermeier, K. Ollefs, F. Wilhelm, A. Rogalev, S. Lebegue, A. L. Da Rosa, and A. Ney, Phys. Rev. B 85, 235203 (2012).

${ }^{16}$ Z. Xu, Q.-R. Zheng, and G. Su, Phys. Rev. B 85, 075402 (2012); S. Haffad, M. Samah, and G. Cicero, Phys. Rev. B 85, 165207 (2012).

${ }^{17}$ R. M. Sheetz, I. Ponomareva, E. Richter, A. N. Andriotis, and M. Menon, Phys. Rev. B 80, 195314 (2009).

${ }^{18}$ G. Shen, B. Liang, X. Wang, H. Huang, D. Chen, and Z. L. Wang, ACS Nano 5, 6148 (2011); P. Schio, F. Vidal, Y. Zheng, J. Milano, E. Fonda, D. Demaille, B. Vodungbo, J. Varalda, A. J. A. de Oliveira, and V. H. Etgens, Phys. Rev. B 82, 094436 (2010); X. Duan, J. Wang, and C. M. Lieber, Appl. Phys. Lett. 76, 1116 (2000); Z. Huo, C.-K. Tsung, W. Huang, X. Zhang, and P. Yang, Nano Lett. 8, 2041 (2008).

${ }^{19}$ G. Kresse and D. Joubert, Phys. Rev. B 47, 558 (1993); G. Kresse and J. Furthmuller, Phys. Rev. B 54, 11169 (1996).

${ }^{20}$ J. P. Perdew, K. Burke, and M. Erzenhoff, Phys. Rev. Lett. 77, 3865 (1996).

${ }^{21}$ M. Toyoda, H. Akai, K. Sato, and H. K. Yoshida, Physica B 376, 647 (2006).

${ }^{22}$ Y. Li, R. Deng, W. Lin, Y. Tian, H. Peng, J. Yi, B. Yao, and T. Wu, Phys. Rev. B 87, 155151 (2013).

${ }^{23}$ G. M. Dalpian and S.-H. Wei, Phys. Rev. B 72, 115201 (2005).

${ }^{24}$ P. E. Blochl, Phys. Rev. B 50, 17953 (1994).

${ }^{25}$ M. Ishii, S. Komuro, T. Morikawa, and Y. Aoyagi, J. Appl. Phys. 89, 3679 (2001).

${ }^{26}$ K. Sato, L. Berqqvist, J. Kudrnovsky, P. H. Dederichs, O. Eriksson, I. Turek, B. Sanyal, G. Bouzerar, H. K. Yoshida, V. A. Dinh, T. Fukushima, H. Kizaki, and R. Zeller, Rev. Mod. Phys. 82, 1633 (2010); K. Sato, W. Schweika, P. H. Dederichs, and H. Katayama-Yoshida, Phys. Rev. B 70, 201202 (2004).

${ }^{27}$ K. Sato, T. Fukushima, and H. K. Yoshida, Jpn. J. Appl. Phys., Part 2 46, L682 (2007); T. Fukushima, K. Sato, H. K. Yoshida, and P. H. Dederichs, Jpn. J. Appl. Phys., Part 2 45, L416 (2006).

${ }^{28}$ S. Geburt, D. Stichtnoth, S. Muller, W. Dewald, C. Ronning, J. Wang, Y. Jiao, Y. Y. Rao, S. K. Hark, and Q. Li, J. Nanosci. Nanotechnol. 8, 244 (2008).

${ }^{29}$ A. Janotti and C. G. Van de Walle, Phys. Rev. B 76, 165202 (2007).

${ }^{30}$ K. Sato and H. Katayama-Yoshida, Jpn. J. Appl. Phys., Part 2 40, L334 (2001).

${ }^{31}$ K. Sato and H. K. Yoshida, Jpn. J. Appl. Phys., Part 2 39, L555 (2000).

${ }^{32}$ M. Ungureanu, H. Schmidt, H. V. Wenckstern, H. Hochmuth, M. Lorenz, M. Grundmann, M. F. Morariu, and G. Gntherodt, Thin Solid Films 515, 8761 (2007)

${ }^{33}$ C. G. Vande Walle, Phys. Rev. Lett. 85, 1012 (2000); X. Wu, D. Vanderbilt, and D. R. Haman, Phys. Rev. B 72, 035105 (2005).

${ }^{34}$ P. M. Krstajic, F. M. Peeters, A. Ivanov, V. Fleurov, and K. Kikoin, Phys. Rev. B 70, 195215 (2004)

${ }^{35}$ H. K. Yoshida, K. Sato, T. Fukushima, M. Toyoda, H. Kizaki, V. A. Dinh, and P. H. Dederichs, Phys. Status Solidi A 204, 15 (2007). 